\title{
VDI
}

\section{REIHE 18}

MECHANIK/

BRUCHMECHANIK

Fortschritt-

Berichte VDI

Lars Kanzenbach, M. Sc.,

Chemnitz

NR. 357

Experimentell-numerische

Vorgehensweise zur

Entwicklung von Probe-

körper-Setups für die

Charakterisierung

technischer Elastomere

\section{BAND}

$1 \mid 1$

VOLUME

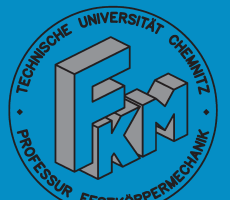

Berichte aus der

Professur Festkörpermechanik

Technische Universität Chemnitz 


\title{
Experimentell-numerische Vorgehensweise zur Entwicklung von Probekörper-Setups für die Charakterisierung technischer Elastomere
}

\author{
Von der Fakultät für Maschinenbau der \\ Technischen Universität Chemnitz \\ genehmigte
}

\section{Dissertation}

zur Erlangung des akademischen Grades Doktoringenieur

(Dr.-Ing.)

vorgelegt

von Lars Kanzenbach M. Sc.

geboren am 30. Januar 1989 in Prenzlau

eingereicht am 21. August 2019

Gutachter:

Prof. Dr.-Ing. habil. Jörn Ihlemann

Prof. Dr.-Ing. Matthias Kröger

Tag der Verteidigung: 29. November 2019 
Persistente URN: http://nbn-resolving.de/urn:nbn:de:bsz:ch1-qucosa2-365142 


\section{VDI}

REIHE 18

MECHANIK/

BRUCHMECHANIK
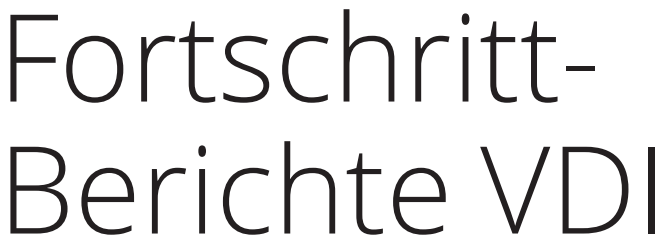

Lars Kanzenbach, M. Sc.,

NR. 357

Experimentell-numerische

Vorgehensweise zur

Entwicklung von Probe-

körper-Setups für die

charakterisierung

technischer Elastomere

BAND

$1 \mid 1$

VOLUME 


\section{VDI verlag}

Kanzenbach, Lars

\section{Experimentell-numerische Vorgehensweise zur Entwicklung von Probekörper-Setups für die Charakterisierung technischer Elastomere}

Fortschritt-Berichte VDI, Reihe 18, Nr. 357. Düsseldorf: VDI Verlag 2021.

158 Seiten, 110 Bilder, 3 Tabellen.

ISBN 978-3-18-335718-5, ISSN 0178-9457
62,00 EUR/VDI-Mitgliederpreis: 55,80 EUR

Für die Dokumentation: Zug-/Druckmessungen - Probekörper-Setup - Probekörper Optimierung -

Schermessungen - Schervorrichtung - homogener Messbereich - Gummiwerkstoffe - Gummi-Phänomenologie

Keywords: Tension-/compression tests - specimen-setup - specimen optimization - shear tests -

shear device - homogeneous measuring zone - rubber materials - rubber phenomenology

Für die Materialcharakterisierung und Parameteridentifikation von technischen Elastomeren werden homogene Probekörper benötigt. Eine besonders wichtige Beanspruchungsart ist dabei der einachsige Zug/Druck. Für Versuche dieser Art findet die Standard-Hantel Anwendung. Allerdings lässt sich hier schon bei geringen Druckbelastungen ein inhomogener Messbereich detektieren. Ein Ziel dieser Arbeit besteht in der Entwicklung eines neuen und verbesserten Probekörpers, der für hochpräzise Zug-/Druckversuche geeignet ist. Darüber hinaus soll der Bereich der maximal erreichbaren Stauchung signifikant erhöht werden. Durch ein spezielles Design der Halterungsgeometrie kann sowohl ein homogenes Verzerrungsfeld erreicht als auch eine hohe Knickstabilität gewährleistet werden. Mit dem entwickelten Probekörper-Setup lassen sich dann eine Vielzahl phänomenologischer Eigenschaften von technischen Elastomeren vorzugsweise bei extremen Stauchungen (bis zu $70 \%$ ) untersuchen.

\section{Bibliographische Information der Deutschen Bibliothek}

Die Deutsche Bibliothek verzeichnet diese Publikation in der Deutschen Nationalbibliographie;

detaillierte bibliographische Daten sind im Internet unter www.dnb.de abrufbar.

Bibliographic information published by the Deutsche Bibliothek (German National Library)

The Deutsche Bibliothek lists this publication in the Deutsche Nationalbibliographie

(German National Bibliography); detailed bibliographic data is available via Internet at www.dnb.de. 


\section{Vorwort}

Die vorliegende Arbeit entstand während meiner Tätigkeit als wissenschaftlicher Mitarbeiter an der Professur Festkörpermechanik der Technischen Universität Chemnitz.

Zuallererst möchte ich mich bei meinem Doktorvater Herrn Prof. Dr.-Ing. habil. Jörn Ihlemann für die Betreuung und Ermöglichung dieser Arbeit bedanken. Neben der Vielzahl an fachlichen Gesprächen hat seine individuelle Förderung und Unterstützung maßgeblich zum Erfolg dieser Arbeit beigetragen. Unsere Zusammenarbeit war auch gewinnbringend für meine persönliche Entwicklung in den vergangenen elf Jahren.

Weiterhin möchte ich mich bei Herrn Prof. Dr.-Ing. Matthias Kröger für das Interesse und die Übernahme des Zweitgutachtens dieser Arbeit bedanken.

Ein weiterer Dank gilt Herrn Dr.-Ing. habil. Martin Stockmann für die gute Zusammenarbeit und die fachlichen Diskussionen gerade im Bereich der experimentellen Mechanik.

Zudem möchte ich mich bei den Mitarbeitern der Professur Festkörpermechanik für die anregenden Diskussionen und die angenehme Arbeitsatmosphäre bedanken. In besonderer Weise gilt das für die Kollegen Sören Gelke, Clemens Schlomka, Dr.-Ing. Thomas Lehmann und Dr.-Ing. Ralf Landgraf.

Ein weiterer Dank richtet sich an die Projektpartner Vibracoustic SE \& Co. KG und Freudenberg Technology Innovation SE \& Co. KG für die gemeinschaftliche Zusammenarbeit und die finanzielle Unterstützung dieses Projektes. Stellvertretend möchte ich mich bei Herrn Dr.-Ing. Rainer Klauke und Dr.-Ing. Christoph Naumann für die zahlreichen Projektbesprechungen bedanken, die zum Gelingen dieser Arbeit beigetragen haben.

Ein besonderer Dank richtet sich auch an Liliana Stoll für das Korrekturlesen dieser Arbeit.

Meine besondere Wertschätzung und mein Dank gilt meinen Eltern Gerno und Sonnlind Kanzenbach. Ich danke Euch, dass Ihr stets hinter mir steht, einen großen familiären Rückhalt bildet und für mich betet! Bedanken möchte ich mich auch bei meinen drei Geschwistern Anika, Lydia und Arne.

Mein größter Dank gilt allerdings meinem Herrn und Retter Jesus Christus. Ich habe ihm so vieles in meinem Leben zu verdanken. Besonders denke ich an seine Kraft, Zuversicht und Freude, die er mir immer wieder schenkt. Ich bin dankbar für seine Treue und die wunderbare Führung in den letzten Jahren.

Lars Kanzenbach 
Die Furcht des Herrn ist der Anfang der Weisheit;

sie macht alle einsichtig, die sie befolgen.

Sein Ruhm bleibt ewiglich bestehen.

Psalm 111,10 


\section{Inhaltsverzeichnis}

$\begin{array}{lll}\text { Abkürzungs- und Symbolverzeichnis } & \text { VII }\end{array}$

$\begin{array}{lll}\text { Kurzfassung } & \text { XI }\end{array}$

$\begin{array}{lll}\text { Abstract } & \text { XII }\end{array}$

1 Einleitung 1

1.1 Motivation der Arbeit . . . . . . . . . . . . . . . . . . 1

1.2 Zielsetzung und Einordnung der eigenen Arbeit . . . . . . . . . . . . . . . 3

2 Grundlagen 5

2.1 Grundbegriffe der Kontinuumsmechanik . . . . . . . . . . . . . . 5

2.1 .1 Tensoralgebra . . . . . . . . . . . . . . . . 5

2.1.2 Verzerrungs- und Spannungstensoren . . . . . . . . . . . . 8

2.1.3 Trennung von Gestalt- und Volumenänderung . . . . . . . . . . . . 10

2.1.4 Hyperelastische Stoffgesetze mit quasi-inkompressibler Formulierung 11

2.2 Stand der Technik zu Elastomer-Probekörpern . . . . . . . . . . . . . . 13

2.2.1 Probekörper für einachsige Zug-/Druckversuche . . . . . . . . . . 13

2.2 .2 Probekörper für biaxiale Versuche . . . . . . . . . . . . . . . 14

2.2 .3 Scherprobekörper . . . . . . . . . . . . . . . . . . . . 15

3 Probekörperdesign für hochpräzise Zug-/Druckmessungen 18

3.1 Motivation für die Entwicklung kombinierter Zug-/Druckprobekörper . . . . 18

3.2 Analytische Untersuchungen zur Knickstabilität von Stäben und Balken . . 19

3.2.1 Differentialgleichung der Balkenschwingung . . . . . . . . . . . 19

3.2.2 Störkraftuntersuchungen am Euler-IV-Druckstab . . . . . . . . . . . 21

3.2.3 Eigenfrequenzanalyse am Euler-Bernoulli-Balken . . . . . . . . . . 26

3.2.4 Erweiterung der Eigenfrequenzanalyse für viskoelastische Strukturen 30

3.3 FE gestützte Entwicklung eines Zug-/Druckprobekörpers . . . . . . . . . . . 34

3.3.1 Grundidee des Halterungsdesigns . . . . . . . . . . . . . . . . . 34 
3.3.2 Algorithmus zur Berechnung von Halterungsgeometrien . . . . . . . 37

3.3.3 Parameterstudie zur Halterungsberechnung . . . . . . . . . . . . . . 38

3.3.4 Fehler- und Instabilitätsmaße . . . . . . . . . . . . . . . . . . 40

3.3.5 Optimierung der Probekörperlänge . . . . . . . . . . . . . . . 45

3.3.6 Formoptimierung der Halterungskontur . . . . . . . . . . . . . 54

3.3.7 Erweiterung des Probekörperdesigns für extreme Stauchungen . . . . 60

3.4 Experimentelle Validierung des neuen Probekörper-Setups . . . . . . . . . . 69

3.4.1 Versuchsaufbau des Probekörper-Setups in der Prüfmaschine . . . . 69

3.4.2 Erweiterte Prüfmaschinensteuerung . . . . . . . . . . . . . . . 71

3.4.3 Voruntersuchungen und Qualitätsprüfungen . . . . . . . . . . 72

3.4.4 Phänomenologische Untersuchung technischer Elastomere . . . . . . 80

4 Entwicklung eines Scherprobekörpers für präzise Schermessungen 88

4.1 Grundlegendes zu einfachen Scherversuchen . . . . . . . . . . . . . . 88

4.2 Motivation für die Verwendung flächiger Scherprobekörper zur Realisierung präziser Schermessungen . . . . . . . . . . . . . . . . . . 90 90

4.3 Grundproblem Lasteinleitung . . . . . . . . . . . . . . . . . . . . . . 90

4.4 Numerische Entwicklung einer Schervorrichtung zur Realisierung präziser Schermessungen . . . . . . . . . . . . . . . . . . . . . . . 92

4.4 .1 Fehler- und Inhomogenitätsmaße . . . . . . . . . . . . . . . . . 92

4.4 .2 Untersuchung von Klemmdesigns . . . . . . . . . . . . . . . . . . 98

4.4.3 Numerische Entwicklung eines Scherprobekörpers mit formschlüssiger Lasteinleitung . . . . . . . . . . . . . . . . . . 102

4.5 Experimentelle Realisierung einer Schervorrichtung mit formschlüssiger Lasteinleitung . . . . . . . . . . . . . . . . . 106

4.5.1 Versuchsaufbau der Schervorrichtung . . . . . . . . . . . . 106

4.5.2 Technologische Überlegungen zur Locherzeugung in Elastomermatten 107

4.5.3 Schermessungen mit Elastomermatten . . . . . . . . . . . . . . . . 108

\section{Anwendungsbeispiele für die entwickelten homogenen Probekörper 113}

5.1 Homogene Standardversuche zur Identifikation von Materialparametern . . 113

5.2 Untersuchung des ratenabhängigen Materialverhaltens von Elastomeren . . 118

5.3 Implementierung des Probekörper-Setups in den Industriealltag . . . . . . . 124

6 Zusammenfassung und Ausblick $\quad 128$

$\begin{array}{ll}\text { A Anhang } & 132\end{array}$

$\begin{array}{ll}\text { Literatur } & 135\end{array}$ 


\title{
Abkürzungs- und Symbolverzeichnis
}

\author{
Abkürzungen \\ DIC Englisch für Digital Image Correlation \\ EPDM Englisch für Ethylene Propylene Diene Monomer \\ FEM Finite-Elemente-Methode \\ FKM Fluorkautschuk \\ GOM Gesellschaft für optische Messtechnik \\ Morph Englisch für Model of Rubber Phenomenology \\ NR Englisch für Natural Rubber \\ PBC Englisch für Periodic Boundary Condition \\ RVE Repräsentatives-Volumen-Element \\ TARRC Englisch für Tun Abdul Razak Research Centre
}

\section{Allgemeine Kennzeichnung}

Symbol Bedeutung

\begin{tabular}{ll}
\hline$X_{a b}$ & Tensorkoeffizienten \\
{$\left[X_{a b}\right]$} & Koeffizientenmatrix \\
$X$ & Tensor nullter Stufe (Skalar) \\
$X_{A}$ & Eigenwerte eines Tensors \\
$\widetilde{X}$ & Größe in der Referenzkonfiguration \\
$\mathrm{d} X$ & Differential (infinitesimal) \\
$\dot{X}$ & Substantielle Zeitableitung von skalaren Größen \\
$\underline{X}, \underline{\underline{X}}$ & Tensor erster und zweiter Stufe \\
$\underline{\underline{\underline{X}}}, \underline{\underline{X}}^{-1}$ & Transponierter und inverser Tensor \\
$\underline{\underline{\underline{X}}}, \underline{\underline{\underline{X}}}$ & Symmetrischer und antisymmetrischer Anteil eines Tensors \\
$\underline{\underline{\underline{X}}}, \underline{\bar{X}}^{\prime}$ & Hydrostatischer und deviatorischer Anteil eines Tensors \\
$\underline{\underline{\underline{X}}}, \underline{\underline{\underline{X}}}$ & Volumen- und Gestaltänderungsanteil eines Tensors \\
$\underline{\underline{\underline{X}}}((\underline{\underline{X}})), S^{-1}((\underline{\underline{X}}))$ & Übertragungsoperator und inverser Übertragungsoperator
\end{tabular}




\section{Produkte}

Symbol Bedeutung

$\underline{\underline{X}} \cdot \underline{\underline{Y}}$

$\underline{\underline{X}} \cdot \underline{\underline{Y}}$

Einfaches Punktprodukt

$\underline{\underline{\underline{X}}} \circ \underline{\underline{Y}}$

Doppeltes Punktprodukt

Dyadisches oder tensorielles Produkt

\section{Skalare}

Symbol

Bedeutung

A

$\alpha$

$\ddot{\alpha}$

$c_{i j}$

$\gamma$

$d$

$\delta_{a b}$

$\delta W^{\mathrm{nc}}$

$\Delta l$

E

$\varepsilon$

$\zeta$

$\eta$

$\eta_{\mathrm{I}}, \eta_{\mathrm{s}}, \eta_{\sigma}$

$\eta_{\mathrm{T}}$

$f$

F

G

$h$

I

$I_{1}, I_{2}, I_{3}$

$\theta$

$J$

$J_{1}, J_{2}, J_{3}$

$\iota$

K

$\kappa$

l

$L$
Fläche

Winkel, Gewichtung

Winkelbeschleunigung

Materialparameter im hyperelastischen Stoffgesetz

Innere Dehnung

Durchmesser

Kronecker-Symbol

Virtuelle Arbeit

Längenänderung

Elastizitätsmodul

Dehnung

Dimensionslose Koordinate

Spezifische Entropie, Viskosität

Lokales Fehlermaß

Globales Fehlermaß

Frequenz, Zielfunktion, Fehlergröße

Kraft

Schubmodul

Höhe

Flächenträgheitsmoment

Hauptinvarianten

Absolute Temperatur

Massenträgheitsmoment

$J$-Invarianten

Größe der Imperfektion

Kompressionsmodul

Instabilitätsmaß

Länge

Lagrangefunktion 
$\lambda$

$m$

M

$\mu$

$n$

$N$

$\nu$

$\xi_{\mathrm{s}}$

$p$

$\varphi$

$\psi$

$q$

Q

$r$

$\rho$

$s$

S

$\bar{\sigma}_{\text {eqv }}$

$t$

T

$\tau$

$u$

$v$

V

w

$\dot{w}$

$\ddot{w}$

$\omega$

$x, y, z$
Eigenwert, Streckung

Masse

Moment

Streckungsfaktor, Reibung

Anzahl der Iterationsschritte

Normalkraft

Querkontraktionszahl

Inhomogenitätsmaß

Hydrostatischer Druck

Eigenform, Winkel

Freie Helmholtz-Energie

Belastungsintensität, generalisierte Koordinate

Querkraft

Radius

Dichte

Schermaß

Störkraft

Mittelwert

Zeit, Dicke

Kinetische Energie

Relaxationszeit

Verschiebung

Verschiebung, Grad der Verzerrung

Potentielle Energie

Verschiebung

Geschwindigkeit

Beschleunigung

Eigenfrequenz

Kartesische Koordinaten

\section{Vektoren}

Symbol

Bedeutung

\begin{tabular}{ll}
\hline$\underline{e}_{a}$ & Basisvektor \\
$\underline{F}$ & Kraftvektor \\
$\underline{n}$ & Normaleneinheitsvektor \\
$\underline{\nabla}$ & Nabla-Operator \\
$\underline{q}$ & Wärmestromdichte \\
$\underline{r}$ & Ortsvektor
\end{tabular}


$\underline{s}$

$\underline{u}$

$\underline{v}$
Spannungsvektor

Verschiebungsvektor

Geschwindigkeitsvektor

\section{Tensoren zweiter Stufe}

Symbol

Bedeutung

$\underline{\underline{b}}$

Linker Cauchy-Green-Tensor

$\underline{\underline{C}}$

Rechter Cauchy-Green-Tensor

$\underline{\underline{D}}$

Tensor der Formänderungsgeschwindigkeit

$\stackrel{\gamma}{=}$

Greenscher Verzerrungstensor

Almansischer Verzerrungstensor

$\underline{\underline{F}}$

Deformationsgradient

$\underline{\underline{h}}$

Eulerscher Hencky-Tensor

H

Lagrangescher Hencky-Tensor

$\underline{\underline{I}}$

Einheits- oder Metriktensor

$\underline{\underline{L}}$

Geschwindigkeitsgradient

$\underline{\underline{\sigma}}$

Cauchy-Spannungstensor

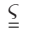

$\underline{\underline{T}}$

Differenz zweier Spannungstensoren

1. Piola-Kirchhoff-Spannungstensor

2. Piola-Kirchhoff-Spannungstensor

Rechter Strecktensor

Linker Strecktensor

$\underline{\underline{V}}$

Wirbeltensor 


\section{Kurzfassung}

Für die Materialcharakterisierung und Parameteridentifikation von technischen Elastomeren werden homogene Probekörper benötigt. Eine besonders wichtige Beanspruchungsart ist dabei der einachsige Zug/Druck. Für Versuche dieser Art findet die Standard-Hantel Anwendung, die für kombinierte Zug-/Druckversuche geeignet ist. Allerdings lässt sich hier schon bei geringen Druckbelastungen ein inhomogener Messbereich detektieren. Ein Ziel dieser Arbeit besteht in der Entwicklung eines neuen und verbesserten Probekörpers, der für hochpräzise Zug-/Druckversuche geeignet ist. Im Gegensatz zur Standard-Hantel wird der für Messungen zugänglich gemachte homogene Messbereich deutlich verbessert. Darüber hinaus soll der Bereich der maximal erreichbaren Stauchung signifikant erhöht werden. Der Probekörper selbst weist dabei eine verhältnismäßig einfache Hantelgeometrie mit verlängertem Mittelteil auf. Durch ein spezielles Design der Halterungsgeometrie kann sowohl ein homogenes Verzerrungsfeld erreicht als auch eine hohe Knickstabilität gewährleistet werden. Die Grundidee besteht dabei darin, dass der Probekörper mit zunehmender Stauchung immer weiter mit der Halterungsgeometrie in Kontakt tritt und dadurch seine knickgefährdete Länge reduziert wird. Mit Hilfe eines speziellen Halterungsalgorithmus kann eine neue, verbesserte Halterungsgeometrie berechnet werden. Mit dem entwickelten Probekörper-Setup (bestehend aus Hantel- und Halterungsgeometrie) lassen sich dann eine Vielzahl phänomenologischer Eigenschaften von technischen Elastomeren wie PayneEffekt, Mullins-Effekt, Erholungs- und Relaxationsverhalten vorzugsweise bei extremen Stauchungen (bis zu $70 \%$ ) untersuchen.

Ein weiteres Ziel dieser Arbeit besteht in der Entwicklung eines Scherprobekörpers zur Realisierung präziser Schermessungen. Das Design soll dabei auf einem flächigen Probekörper (Elastomermatte) beruhen, um Alterungsuntersuchungen, Untersuchungen mit faserverstärkten Materialien und Versuche mit Vorreckungen realisieren zu können. Im Gegensatz zu herkömmlichen Scherprobekörpern soll dabei auf eine stoffschlüssige Verbindung mittels Kleben oder Anvulkanisieren aufgrund von Materialirritationen oder Schrumpf verzichtet werden. Im Rahmen dieser Arbeit wurde diesbezüglich ein spezielles Fixierdesign mit Stiften entwickelt, welches zur Ausbildung nahezu homogener Scherdeformationen führt. Damit lassen sich eine Vielzahl wichtiger Eigenschaften bei einer annähernd homogenen Scherdeformation untersuchen.

\section{Schlagworte}

Zug-/Druckmessungen, Probekörper-Setup, Probekörper Optimierung, Schermessungen, Schervorrichtung, homogener Messbereich, Gummiwerkstoffe, Gummi-Phänomenologie 


\section{Abstract}

Homogeneous test specimens are required for material characterization and model parameter identification. An important kind of loading is uniaxial tension/compression. For this, a standard dumbbell is available for combined tension-compression tests. But even for small compressive strains the standard dumbbell leads to an inhomogeneous stress state in the measuring zone. One aim of this work is the development of a new test specimen, which is suitable for high-precision tension/compression tests. In comparison to the standard dumbbell the homogeneity in the measuring zone is significantly improved. Furthermore, the range of maximal compression is increased substantially. The test specimen itself consists of a slender dumbbell structure. By a special design of the mounting geometry, homogeneous stress and strain fields as well as a high stability can be achieved. For an increasing compression, the test specimen comes into contact with the mounting geometry and the critical length is reduced. By means of dynamic analysis, the mounting geometry was calculated and optimized. This method is a powerful tool for developing new mounting geometries, by taking into account both the stability and the homogeneity characteristic. With the developed specimen-setup (consisting of dumbbell and mounting geometry), the phenomenological characteristics of rubber like Payne effect, Mullins effect, recovery and relaxation behavior can be investigated up to a compressive strain of $70 \%$.

Another aim of this work is the development of a shear specimen, which enables precision shear measurements for large shear values. The design is based on a planar test specimen (rubber mat) in order to enable ageing tests, tests with fibre-reinforced materials and tests with pre-stretching. In contrast to other shear specimens, a material-locking connection by gluing or vulcanizing sould be avoided in consequence of material irritation or shrinkage. For this, a special fixing design was developed, which enables a uniform initiation of shear deformation for different rubber thicknesses. Finally, the new shear specimen enables the investigation of typical rubber properties.

\section{Keywords}

Tension-/compression tests, specimen-setup, specimen optimization, shear tests, shear device, homogeneous measuring zone, rubber materials, rubber phenomenology 